\title{
(6) OPEN ACCESS \\ Impact of two-step urine culture ordering in the emergency department: a time series analysis
}

\author{
Amanda Stagg, ${ }^{1}$ Haydon Lutz, ${ }^{2}$ Sakshi Kirpalaney, ${ }^{2}$ John Justin Matelski, ${ }^{3}$ \\ Adam Kaufman, ${ }^{1}$ Jerome Leis, ${ }^{4,5}$ Janine McCready, ${ }^{1,4}$ Jeff Powis ${ }^{1,4}$
}

\begin{abstract}
'Michael Garron Hospital, Toronto, Ontario, Canada ${ }^{2}$ Royal College of Surgeons in Ireland School of Medicine, Dublin, Ireland

${ }^{3}$ Department of Internal Medicine, Toronto General Hospital, Toronto, Ontario, Canada

${ }^{4}$ Department of Medicine, University of Toronto, Toronto, Ontario, Canada

${ }^{5}$ University of Toronto, Center for Quality Improvement and Patient Safety, Toronto, Ontario, Canada
\end{abstract}

\section{Correspondence to} Dr Jeff Powis, Michael Garron Hospital, Toronto, Ontario, Canada M4C 3E7; jpowi@tegh.on.ca

Received 30 October 2016 Revised 28 March 2017 Accepted 8 April 2017 Published Online First 3 May 2017

\section{SLinked}

- http://dx.doi.org/10.1136/ bmjqs-2017-006793

\section{CrossMark}

To cite: Stagg A, Lutz $\mathrm{H}$,

Kirpalaney $S$, et al.

BMJ Qual Saf

2018;27:140-147

\section{ABSTRACT}

Background Despite evidence against the use of antimicrobials for asymptomatic bacteriuria (ASB), they are frequently prescribed leading to unnecessary adverse events. Prior studies have shown that reducing unnecessary urine cultures (UCS) results in decreased antimicrobial utilisation for ASB. Emergency departments (EDs) submit the largest volume of UCS, yet efforts to limit overordering in this patient setting have had limited success.

Methods A new two-step model of care for urine collection, using a novel UC collection container, was implemented in the ED of a large community hospital. The collection system contains a preservative allowing UCs to be held at room temperature for up to 48 hours before processing. UCs were collected by front-line staff, but only processed in the microbiology lab if requested by ED physicians after clinical assessment.

Results Following implementation there was a decrease in the percentage of weekly ED visits associated with a processed UC $(5.97 \%$ vs $4.68 \%, p<0.001)$, a decrease in the percentage of monthly ED visits requiring a callback for positive urine culture $(1.84 \%$ to $1.12 \%$, $\mathrm{p}<0.001)$ and a decrease in antimicrobial prescriptions for urinary indication among admitted patients $(20.6 \%$ to $10.9 \%, p<0.01)$. There was a false omission rate of $1.35 \%$ (95\% $\mathrm{Cl} 0.7 \%$ to $2.2 \%$ ), yet no identified cases of untreated urinary tract infection (UTI), or significant change in repeat ED visits or ED length of stay.

Conclusions Changing to two-step urine culture ordering in the ED resulted in a decrease in UCS processed, callbacks for positive results and antimicrobial use without evidence of untreated UTIs. This model of care has strong potential to improve the use of hospital resources while minimising detection and inappropriate treatment of ASB.

\section{INTRODUCTION}

Urine cultures (UCs) remain the most overordered clinical microbiological investigation across acute and long-term care institutions. ${ }^{1}$ This excess testing is not only a waste of finite healthcare resources, but results in the overdetection of asymptomatic bacteriuria (ASB). Overdetection of ASB leads to low-value care, including antimicrobial prescriptions that are of no benefit and potentially harmful to patients. $^{2-5}$ Between $30 \%$ and $60 \%$ of patients with ASB receive unnecessary antimicrobial treatment, which is associated with an eightfold risk of developing Clostridium difficile infection and is one of the leading causes of unnecessary antibiotic use driving antimicrobial resistance. ${ }^{2}{ }^{6-8}$

Emergency departments (EDs) submit the largest volume of UCs in the hospital, yet improvement efforts to limit overordering in this patient setting have had limited success. ${ }^{139}$ The fast paced environment of the ED favours the preemptive collection of UCs for nonspecific indications, to facilitate subsequent patient management. ${ }^{10}$ Any intervention to address overordering in the ED must also guard against underinvestigation and undertreatment of patients who are acutely ill and diagnostically undifferentiated on presentation.

Education in the form of diagnostic and treatment algorithms to promote appropriate ordering of UCs and antibiotic prescribing for symptomatic urinary tract infections (UTIs) have had limited success and require significant resources to sustain. ${ }^{11-13}$ Even when the pretest probability of UTI is low, positive UC results greatly influence diagnosis and the decision to treat with antibiotics. ${ }^{4} 814$ 
A prior proof-of-concept study among hospitalised inpatients demonstrated that when positive UCs are no longer routinely reported, antimicrobial use for ASB drops significantly. ${ }^{14}$ Processing, yet not reporting UCs, however, still requires substantial microbiology resources and does not optimise cost savings. A process that allows for UCs to be collected preemptively, yet held until clinical probability of UTI can be determined, would be the preferred approach. The challenge in creating such a model has been that delaying the processing of urine specimens can reduce specimen quality, increase bacterial overgrowth and ultimately lead to false-positive results. ${ }^{1}$ In addition, due to the volume of UCs submitted from the ED, refrigerating held urine specimens to limit bacterial overgrowth is not a feasible strategy.

Recently, a novel urine collection system containing a preservative has been demonstrated to allow UCs to be held at room temperature for up to 48 hours while maintaining valid results. ${ }^{15}$ This new collection system allows urine to be pre-emptively collected in specimen containers with preservative, yet held until the clinical need for the UC can be determined following a physician assessment. We hypothesised that this new two-step model of UC ordering can reduce treatment of ASB through decreased processed UCs in the ED. We implemented this new model of care in the ED at our community hospital in the fall of 2014 and performed a time series analysis to evaluate the efficacy, safety and costs associated with this intervention.

\section{METHODS}

\section{Setting}

The study was completed at a 515-bed acute care community teaching hospital in a large urban centre, with an ED that has approximately 73000 visits annually. The department employs 150 staff and 40 emergency physicians. At baseline, approximately 90 UCs per month were sent to the laboratory, which represent, on average, $53 \%$ of the urine cultures ordered throughout the organisation. All UC results are automatically reported in the patient's electronic medical record (EMR). The ED has a daily, physician-led callback system for all positive microbiology results from patients discharged from the ED.

Prior to the implementation of our quality improvement initiative a $24 \%$ decrease in UCs collected in the ED was achieved using front-line ownership (FLO). ${ }^{10}$ The FLO intervention took place from July to December, 2013, and involved active engagement of front-line staff to determine root causes and develop practical solutions to optimise UC ordering rates. Findings during FLO engagement noted that UCs were in poor compliance with nursing guidelines, were pre-emptively being done to facilitate flow, or were being sent to the lab so that urinalysis results would be imputed into the EMR rather than having to be done through point-of-care (POC) testing. These findings led to the development of the change concept and subsequently change ideas used for this study. UC ordering rates in the ED have slowly trended down following the FLO engagement.

\section{Implementation of intervention}

In May of 2015, the UC containers in our ED were replaced by the BD Vacutainer plus urine C\&S preservative tubes system (New Jersey, USA) and the process for ordering a UC was modified. The UC ordering process before and after intervention is displayed graphically in figure 1 . The intervention involved a two-step ordering process for UCs in the ED. First, any nurse, through a medical directive, could submit urine in a collection container with preservative directly to the laboratory by ordering a 'Step 1: Collect Urine'. The 'Step 1' urine order included an order for urinalysis as well as UC. This was done to promote the availability of urinalysis results at the time of clinician assessment and facilitate improved turnaround time (TAT) for urinalysis in the laboratory. The UCs from the 'Step 1' were sent to the local laboratory and stored in wired racks marked and held for the day of the week in which they were collected. The laboratory also organised the cultures based on electronic ordering number, as a means to increase efficiency when needing to locate the specimen from the racks. Following clinical assessment by the ED physician, if the UC was required, a second order was entered for 'Step 2: Process Urine'. Receipt of a 'Step 2' order prompted the laboratory technologist to send the urine for culture and susceptibility testing at the microbiology laboratory. Each day, a technologist would discard urine cultures that were over 48 hours old, allowing the ED physicians up to 48 hours to request the urine culture be processed. Even if a nurse had not initially collected a urine culture, a physician ordering a 'Step 2: Process Urine' would lead to a nurse collecting the urine, which would be automatically processed for culture by the laboratory.

\section{Training}

The vendor of the UC collection system containing preservative and the ED nurse educator spent 2 weeks training ED and laboratory staff on the new two-step ordering process. Implementation was systematic in its design; once staff had been trained, a 2-week question and answer period was initiated. This allowed a grace period for the departments to work out any foreseeable issues or concerns brought forward by the staff. During this period the most common questions related to how the ED clinical team could confirm a urine culture had been collected or processed on a particular patient and whether the new process would lead to delays in urine culture TAT time. Vendor costs related to providing an expert trainer were captured. 


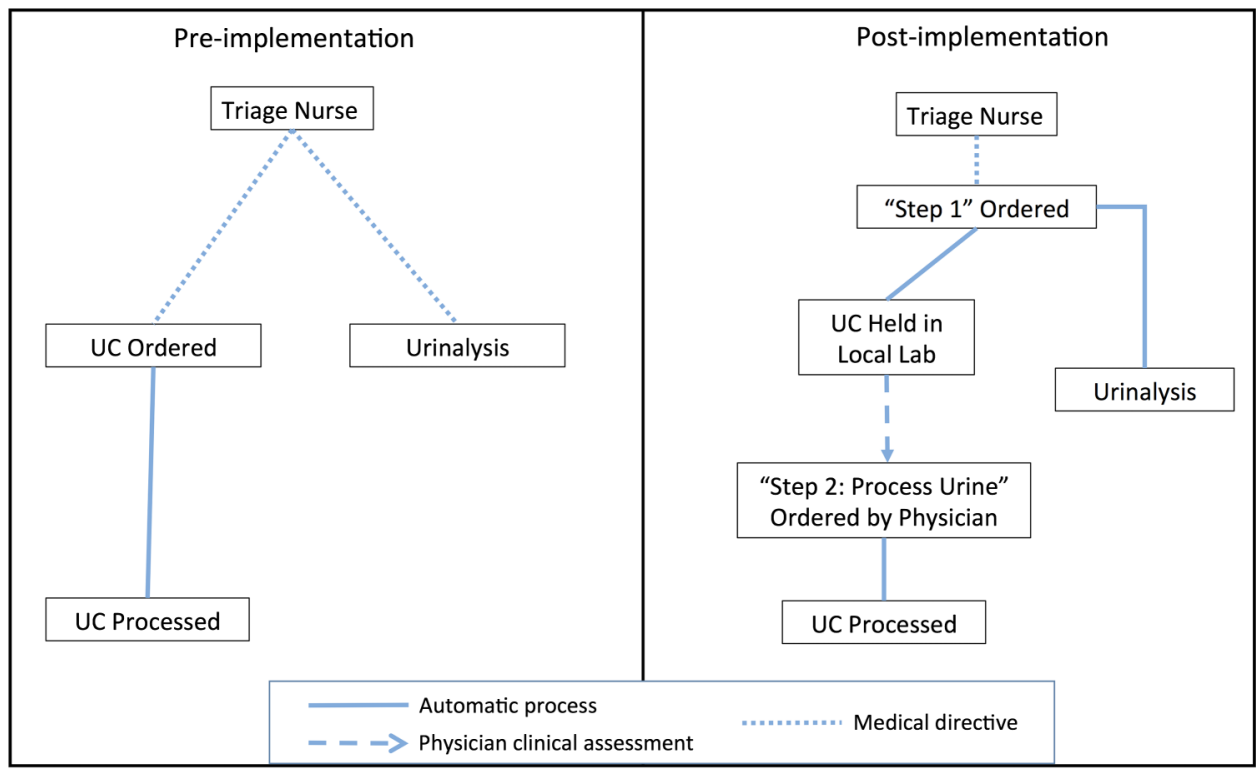

Figure 1 Process map depicting the change in model of care for urine culture (UC) ordering in the emergency department after intervention implementation. The above figure represents the UC and urinalysis ordering processes in the emergency department before and after introduction of the new model of care for UC ordering. Lab, laboratory.

\section{Co-intervention}

In the process of implementation, a key barrier was identified to the successful adoption of the new model of care for UC ordering. The ED physicians noted that their clinical assessment about whether to order 'Step 2: Process Urine' would be improved if the urinalysis results would be available on the EMR rather than having to be done through POC testing, printed and placed on the patient's paper chart. The existing process was to perform POC testing and then submit the specimen to the laboratory for confirmation of urinalysis results and microscopy. To address this barrier and increase ED engagement in the project, the team worked to reduce urinalysis TAT in the laboratory. The major delay in the reporting of urinalysis results was due to abnormal urinalysis results automatically triggering a microscopic evaluation in the laboratory. The laboratory uncoupled urinalysis from microscopy and allowed for autoverification of urinalysis results with subsequent immediate release to the EMR.

\section{Outcome and process measures}

An interrupted time series analysis was performed to evaluate the impact of the new model of care for UC ordering using collection containers with preservative on incidence of UCs processed in the ED. All catheter and non-catheter urine culture specimens collected from adult patients in the ED from the weeks starting 6 January 2014-18 May 2015 (baseline) to 25 May 2015-28 December 2015 (intervention period) were included in the analysis. Monthly ED patient demographics were extracted from the hospital information system.
The primary outcome measure was the percentage of ED visits where a UC was processed expressed as the ED urine culture percentage. A processed UC was defined as a urine specimen that was sent from the ED for culture and processed by the microbiology laboratory. Weekly UCs processed were extracted from the laboratory information system while the number of weekly ED visits was determined from the hospital information system.

Secondary outcome measures included the monthly percentage of ED visits requiring a callback for positive urine cultures, urinalysis TAT and antimicrobial utilisation among patients admitted to the hospital initially or within 7 days of their initial ED visit. Monthly ED visits requiring a callback was determined through extracted positive urine culture results from the laboratory electronic database from patients seen and subsequently discharged from the ED during the study period. Systemic antimicrobial utilisation (non-topical) was determined through manual chart audit of all patients with a urine culture in July 2014 (preintervention) and among patients with 'Step 1' urine in July 2015 (postintervention). Antimicrobials were deemed to be for a urinary indication only if this was documented in the patient's chart. Physician costs associated with callbacks were estimated using the standard hourly rate based on provincial funding of $\$ 170$ and the mean time of 19.6 min required to perform a callback. Lastly, costs related to the purchase of the new urine collection system were tracked monthly following implementation as were laboratory costs related to processing of urine cultures.

Process measures included the number of ED staff trained in the new UC ordering process as 
documented by sign-off of department supervisors, and the monthly number of UCs sent to the laboratory but not processed and the monthly number of urinalyses ordered.

\section{Balancing measures}

To assess for any underinvestigation or undertreatment resulting from the new model of care for UC ordering, we tracked the monthly average 7-day repeat $\mathrm{ED}$ visit rate before and after implementation of the intervention. This time window was specifically selected given the expected time to symptom resolution or deterioration related to an untreated UTI. ${ }^{16}$ We also performed a retrospective chart review of all consecutive patients with UCs that were sent to the laboratory without being processed during a randomly selected month of the intervention period (July, 2015) to determine our false omission rate (patients with documented symptoms of UTI who may have benefited from the diagnostic information obtained through processing of their urine culture). The number of monthly urinalyses was monitored to ensure that the coupling of urinalysis to the 'Step 1' urine culture order did not increase urinalysis rates. Finally, to assess for delays in patient flow related to the new UC ordering system, the monthly ED length of stay in hours was measured.

\section{Analysis}

The goal of the statistical analysis was to estimate the effect of the intervention with respect to the primary and secondary outcomes (ED urine culture percentage, monthly percentage of ED visits requiring a callback for positive urine culture and urinalysis TAT), while admitting the possibility of temporal trends, seasonal effects and autocorrelation. We adopted a regression discontinuity approach using generalised linear models to this end. It was not possible to know the best fitting model a priori. To circumvent this, we fit several models for each outcome (eg, with/without monthly effects, with/without temporal trends, etc) and selected the most appropriate on the basis of having the lowest AIC statistic. Additionally, we required that our final models did not evidence statistically significant autocorrelation as defined by a residual Box-Pierce test $\mathrm{p}$ value less than or equal to 0.05 . For each of the three final models we reported the estimated (adjusted) intervention effects with 95\% CIs with Wald test $\mathrm{p}$ values against the null hypothesis of 'zero intervention effect'.

Supplementary analyses were conducted via simple linear regression and a $\chi^{2}$ test of independence. All computations were made using R V.3.0.2.

\section{RESULTS}

Implementation of two-step UC ordering

A total of 121 nurses (RNs and RPNs) and 46 physicians were trained $(66 \%$ and $50 \%$ trained in the first week, respectively) representing $100 \%$ of the clinical staff in the ED. Of the UCs ordered, 19.1\% (95\% CI $18.2 \%$ to $20.0 \%$ ) had a 'Step 2' order from a physician and were processed in the microbiology laboratory, and none of the UCs without a 'Step 2' order was processed.

Average monthly ED patient demographics (preintervention and postintervention) are found in table 1 . There were 100801 patients seen by the ED physician in the baseline period and 44111 in the intervention period. There were more patients in the intervention period with a discharge diagnosis of UTI, site unspecified $(p=0.04)$ and fewer patients with unspecified sepsis $(p=0.03)$ compared with the baseline period.

\section{Outcome measures}

There was a $36.8 \%$ relative reduction in the unadjusted percentage of ED visits with a processed UC after implementation of two-step UC ordering from $6.96 \%$ at baseline to $4.4 \%$ after intervention (figure 2). After model adjustment (table 2), this difference remained significant $(5.97,95 \%$ CI 6.47 to 5.50 vs $4.68,95 \%$ CI 5.16 to $4.24, \mathrm{p}<0.001$ ).

The adjusted monthly percentage of ED visits requiring a callback decreased from 1.84 (95\% CI 1.93 to $1.76)$ to 1.12 (95\% CI 1.23 to 1.03 ) ( $\mathrm{p}<0.001)$. There was a decrease in adjusted urinalysis TAT from $30.55 \mathrm{~min}(95 \% \mathrm{CI} 33.08$ to 28.01$)$ to $12.09 \mathrm{~min}$ (95\% CI 14.53 to 9.64$)(\mathrm{p}<0.001)$ (see table 2).

At baseline (July 2014), 36.6\% (141/385) of patients who had a urine culture were admitted to hospital, of whom $61.0 \%(86 / 141)$ received antimicrobial therapy including $20.6 \%(29 / 141)$ for a

Table 1 Average monthly emergency department (ED) demographics prior to and after intervention

\begin{tabular}{|c|c|c|c|}
\hline Metric & $\begin{array}{l}\text { Preintervention } \\
\mathrm{N}=17\end{array}$ & $\begin{array}{l}\text { Postintervention } \\
n=7\end{array}$ & p Value \\
\hline Admissions to ED & 5929 & 6302 & 0.001 \\
\hline \multicolumn{4}{|l|}{ Age distribution, years } \\
\hline$<18$ & $13.7 \%$ & $12.4 \%$ & \multirow[t]{3}{*}{$<0.001$} \\
\hline $18-65$ & $63.7 \%$ & $64.3 \%$ & \\
\hline$>65$ & $22.6 \%$ & $23.4 \%$ & \\
\hline \multicolumn{4}{|l|}{ Gender } \\
\hline Male & $47.5 \%$ & $48.2 \%$ & \multirow[t]{2}{*}{0.009} \\
\hline Female & $52.5 \%$ & $51.8 \%$ & \\
\hline \multicolumn{4}{|c|}{ Main discharge diagnosis } \\
\hline $\begin{array}{l}\text { Urinary tract } \\
\text { infection, site not } \\
\text { specified }\end{array}$ & $1.55 \%$ & $1.79 \%$ & 0.045 \\
\hline Fever, unspecified & $0.91 \%$ & $0.86 \%$ & 0.297 \\
\hline Sepsis, unspecified & $0.22 \%$ & $0.16 \%$ & 0.029 \\
\hline $\begin{array}{l}\text { Delirium, } \\
\text { unspecified }\end{array}$ & $0.11 \%$ & $0.12 \%$ & 0.860 \\
\hline $\begin{array}{l}\text { Admitted from ED to } \\
\text { hospital }\end{array}$ & $13.0 \%$ & $12.0 \%$ & $<0.001$ \\
\hline
\end{tabular}

Values represent monthly means. 


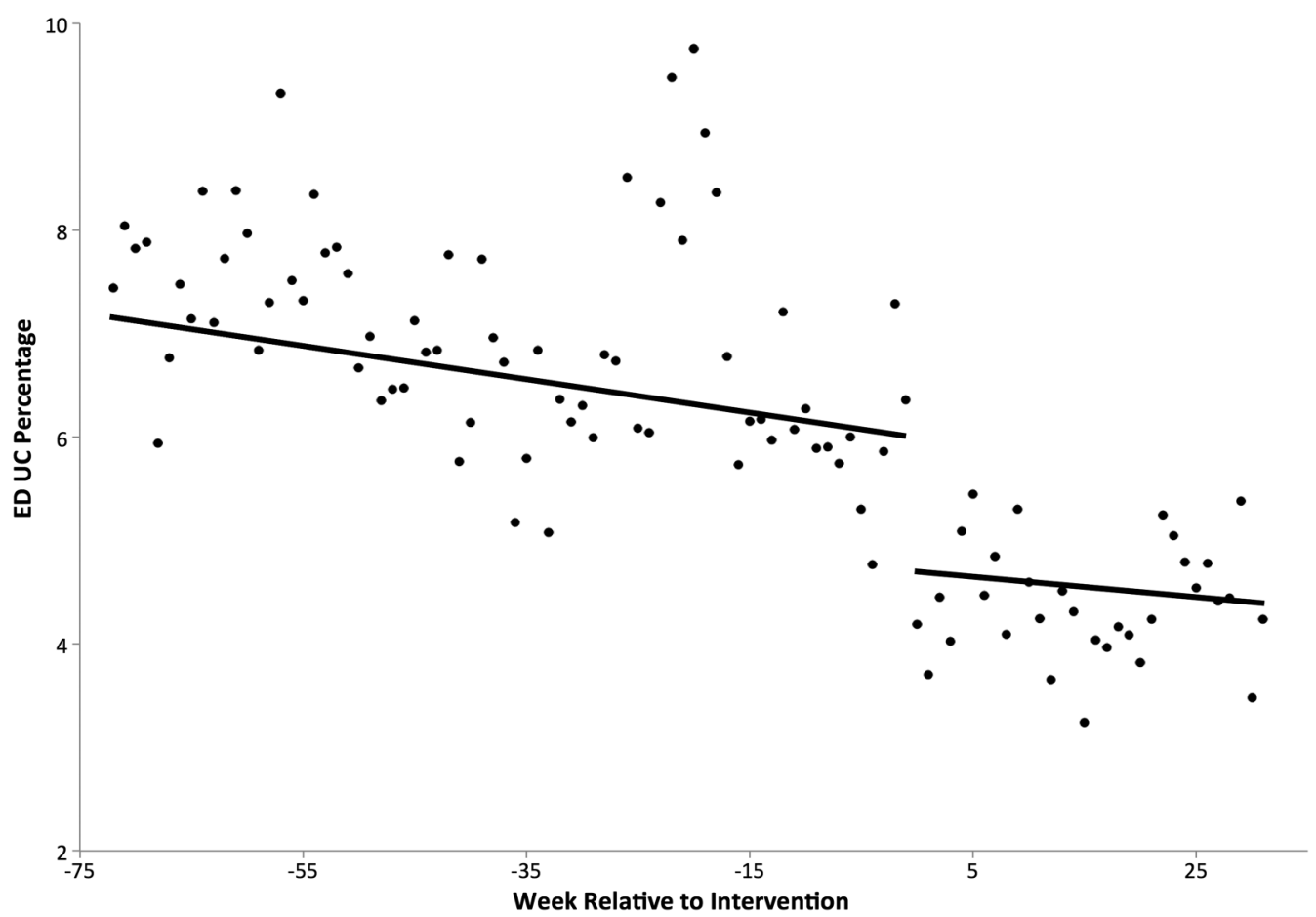

Figure 2 Emergency department (ED) urine cultures (UCS) percentage before and after intervention. This figure depicts the stepwise decline in weekly ED UC percentage after introduction of the new model of care for UC ordering from the preintervention phase to the intervention phase. The solid line represents the linear trend for each phase.

Table 2 Primary and secondary outcomes after introduction of a new model of urine culturing in the emergency department (ED)

\begin{tabular}{lccccc}
\hline Outcome & Baseline & Intervention & Intervention effect (95\% Cl) & p Value & $\begin{array}{l}\text { Box-Pierce } \\
\mathbf{p} \text { value }\end{array}$ \\
\hline Weekly ED urine culture percentage & 5.97 & 4.68 & $-1.29(-0.34$ to -2.23$)$ & $<0.001$ & 0.70 \\
Monthly ED callback percentage & 1.84 & 1.12 & $-0.72(-0.53$ to -0.90$)$ & $<0.001$ & 0.22 \\
Monthly urinalysis TAT (minutes) & 32.33 & 11.33 & $-21.01(-14.85$ to -27.17$)$ & $<0.001$ & 0.43 \\
\hline
\end{tabular}

This table depicts the main outcomes measured during the intervention after model adjustment.

TAT, turnaround time.

urinary indication. With introduction of two-step UC ordering (July 2015), 27.0\% (330/1220) of patients with urine specimens submitted ('Step 1') were admitted to hospital and among these 330 admitted patients, there was a significant reduction in both overall antimicrobial therapy $(162 / 330,49.1 \%$, $\mathrm{p}=0.02)$ driven by a reduction in antibiotics prescribed for urinary indication $(36 / 330,10.9 \%$, $\mathrm{p}<0.01$ ).

\section{Balancing measures}

Introduction of two-step UC ordering led immediately to urine cultures that were collected but not processed at a rate of $15.0 \%$ of $\mathrm{ED}$ visits $(95 \%$ CI $14.4 \%$ to 15.6\%). Although the number of UCs drawn increased, there was no significant change in the rate of urinalysis after intervention implementation (19.1\% of ED visits vs $19.4 \%$ of $E D$ visits) $(p=0.6)$. The baseline average 7-day repeat ED visit percentage was $10.6 \%$ and remained stable at $10.6 \%$ following implementation of the new UC ordering process $(p=0.2)$. The average LOS did not change significantly after intervention (5.4 hours vs 5.1 hours, $\mathrm{p}=0.07$ ).

Over the course of the randomly selected month during the intervention period (July 2015), a total of 1220 patients had their urine collected ('Step 1') in the ED of which 282 were processed and 937 were discarded after 48 hours. Among the 282 patients who had their UC processed the mean time from 'Step 1' to 'Step 2: Process Urine' order was 0.84 hours with an SD of 1.53 hours. Of the specimens $17.8 \%$ had $>2$ hours between 'Step 1: Collect Urine' and 'Step 2: Process Urine'.

Among the 937 patients that did not have their UC processed, 12 patients were identified as potentially having benefited diagnostically from having their UC processed leading to a false omission rate of $1.3 \%$ (95\% CI $0.7 \%$ to $2.2 \%$ ). None of these false omissions were associated with patient harm as all were treated 
empirically with antimicrobials despite not having a UC processed. Ten were admitted to the hospital; one patient was discharged from the ED yet later recalled for a Gram-negative bacteraemia and one patient returned to the ED within 24 hours of their first visit with fever, respiratory failure and Gram-negative bacteraemia. Following antibiotic treatment, all patients improved and were discharged home.

\section{Cost-effectiveness analysis}

The total cost related to purchase of the novel UC collection system on a monthly basis came to $\$ 181.44 \pm 11.44$, with a per capita cost of $\$ 2.88 / 100$ ED visits. Vendor training costs associated with inservices to educate staff about the new process and novel UC collection system were $\$ 672.00$. There were 0.72 callbacks/100 ED visits prevented, yielding an estimated costs avoidance of $\$ 39.98 / 100$ ED visits. Excluding the initial vendor training costs, the net savings in Canadian dollars are an estimate \$58.81/ 100 ED visits.

\section{DISCUSSION}

Our time series analysis demonstrates that a two-step UC ordering process for the ED results in improved antimicrobial prescribing for UTI as evidenced by a decreased need for patient callbacks for positive urine cultures and a decrease in antibiotic prescriptions for a urinary indication among admitted patients. This model of care was not associated with any increase in repeat ED visits within 7 days and a detailed chart audit revealed only a $1.3 \%$ false omission rate with all patients having been treated empirically despite the lack of a processed urine culture.

Due to the fast paced nature of the ED, educational interventions have had limited success in preventing overordering of UCs particularly if they are perceived as increasing workload. ${ }^{39}$ A redesign in the way UCs are ordered in the ED that maintains efficiency is more likely to achieve and sustain improved UC ordering practices. Recently, a reflex urine culture cancellation protocol where UCs were automatically cancelled if the urinalysis was negative was proposed and externally validated. ${ }^{9}{ }^{17}$ In an observational study, this protocol was predicted to reduce processed UCs by $34.6 \%-39.1 \%$ with only a $1.8 \%-2.3 \%$ false omission rate, similar to the impact of our intervention. In a second external validation, the false omission rate was higher at $4.7 \%$.

The advantage of our two-step ordering process, however, is that the decision to process a UC is based on clinical assessment by a physician rather than the result of urinalysis alone. The diagnosis of a UTI is a clinical diagnosis that requires clinician interaction with a patient to establish their signs and symptoms and cannot be determined based on the results of a urinalysis. In the ED, urinalysis alone in the absence of clinical symptoms is a reasonably sensitive tool yet has poor specificity (43.1\%) with a false omission rate far higher than that seen with our intervention. In addition, asymptomatic pyuria can occur in up to $90 \%$ of elderly patients where processing UCs is likely to yield ASB and potentially prompt unnecessary antimicrobial therapy.

In fact, the prior proof-of-concept study by Leis et al demonstrated the impact of not routinely reporting low-risk UCs unless a physician request is made, on treatment of ASB. Our model of care extends this concept to no longer processing urine specimens routinely unless a separate physician order is received. This intervention ensured that among all UCs collected in the ED, only those that were deemed clinically indicated following a physician assessment were processed resulting in improved stewardship of both antimicrobials and microbiology resources. Among a subset of our study population that was admitted to hospital after their initial ED visit there was a significant reduction in antimicrobial use.

This new two-step model for ordering UCs in the ED was relatively simple to implement and could be scalable across institutions to address overordering of UCs in the ED without the need for ongoing human resource allocation after implementation. Front-line ED physicians did not need to invest any additional effort since they simply ordered a 'Step 2: Process Urine' at the same time as any other investigation. The model requires CPOE to develop an order for 'Step 1: Collect Urine' and 'Step 2: Process Urine' as well as the capacity for the CPOE to interact with the laboratory information system to inform the laboratory of the physician request to process the urine. The preservative-based UC collection system requires training of nursing staff to collect urine specimens using this system. We also improved urinalysis TAT so that these results would be available on the EMR rather than POC at the time the ED physician was assessing the patient.

Although the UC collection system that we used is more expensive than traditional sterile containers, our cost analysis demonstrated that the costs of implementation are more than offset through reductions in UC processing costs and physician time to perform callbacks. This analysis does not take into account additional potential savings in antimicrobial utilisation, or costs associated with adverse events related to excess antimicrobial use. The reduced number of physician callbacks, is a surrogate measure for reduced detection of positive UCs which is known to drive antimicrobial prescribing. ${ }^{14}$ The time savings associated with the reduced callbacks would be an important motivating feature in having ED physicians adopt this new way of ordering UCs in the ED. ED callbacks have been demonstrated to be an important quality intervention leading to reductions in unnecessary patient readmissions. ${ }^{18}$ The impact of ED callback systems triggered on positive microbiology could potentially be 
improved if the utility of microbiology in the ED is associated with high pretest clinical probability to ensure the positive microbiological test has real clinical implications.

The benefit of this intervention also needs to be weighed against any possible patient harm. In our evaluation, we actively looked for evidence of underinvestigation and did not find any difference in terms of repeat visits to the ED. It was only upon chart review of over 1000 patients that we identified that $1.3 \%$ of patients with unprocessed UCs may potentially have benefited diagnostically from having a UC performed. Among these false omissions, all patients were treated empirically based on clear symptoms of infection. In considering the reason for these omissions, it is interesting to note that 10 of 12 patients were admitted patients with UTI where the admitting physician failed to order a 'Step 2: Process Urine.' Further training of inpatient physicians would have helped to address this problem since these physicians would have up to 48 hours to enter a 'Step 2' order.

The two-step model for ordering UCs is designed to respect physician autonomy as the decision to process a particular patient's UC is based on their clinical assessment. As such, diagnostic error will always remain and it would be unrealistic to aim for a zero false omission rate in UCs processed. Taken together, this study suggests that with appropriate awareness and training regarding the two-step UC ordering process, the improvement in antimicrobial prescribing for UTI in the ED resulting from this model of UC ordering, greatly outweighs any risk of patient harm.

\section{Limitations}

This study has several important limitations. First, healthcare providers were engaged in the development of the intervention with awareness of the intervention's goal of reducing UC rates. As a result, it is possible that a decrease in UCs processed resulted from Hawthorne effect among the ED staff, the change in behaviour that occurs when providers know they are being observed. The total number of UCs ordered, however, did not change significantly during the intervention period, suggesting that ordering behaviours remained unchanged while the intervention to not process specimens without a separate physician order was responsible for the decrease observed. Second, our ED functions with a medical directive for nurses to order laboratory investigations prior to physician assessment to facilitate patient flow, which may be associated with higher rates of overordering. The impact of our new model for UC ordering would likely be less significant among institutions lacking a similar directive or with established treatment guidelines for uncomplicated UTIs without UC. Third, the cost-effectiveness analysis performed assumes a specific baseline rate of UC overordering prior to implementation of this novel UC ordering system. Due to our prior FLO work in the ED to reduce UC overordering, our baseline rate may be lower than other institutions' leading to an underestimation of cost avoidances associated with the intervention in comparison. Fourth, differences in the ED patient population between the baseline and intervention period may have affected UC ordering practices; however, these differences may have underestimated the impact of our intervention, as there were more patients in the intervention period with a discharge diagnosis of UTI. Finally, the two-step model for UC ordering relies on the use of a preservative-based urine culture collection system. In our study $17.8 \%$ of processed urine cultures had >2-hour delay between collection and order to process the urine culture. This degree of delay could potentially lead to false-positive urine cultures due to bacterial overgrowth if a preservative-based system was not used.

\section{CONCLUSIONS}

A new model of care for UC ordering in the ED was designed to reduce processing of unnecessary UCs without any associated undertreatment of UTI or delays in patient management. The key to the success of this intervention was the use of a preservative-based urine culturing system that allows for a UC to be drawn and held until the time of a separate physician order to have the UC processed. This two-step model for UC ordering facilitated ED patient flow, while ensuring that UCs were only processed when a physician determined a sufficient pretest probability of a UTI existed. As an important co-intervention, reducing the TAT of the urinalysis so that results would be easily available in the EMR at the time of the ED physician assessment, proved to be crucial in garnering ED physician support for this new model of care.

Acknowledgements The authors thank the ED staff and the laboratory for providing them with valuable insights and energy throughout the quality improvement process.

Contributors All authors made substantial contribution to the project conception, study design, data analysis and drafting of the paper. All authors have approved this version of the paper for publication.

Funding This research received no specific grant from any funding agency, commercial or not-for-profit sectors. The UC system was paid for by hospital and no private funding was received. The company (BD) that manufactures this system did not have any role in the design of the study, the conduct of the study, the collection, management, analysis or interpretation of the data, or the preparation, review, or approval of the manuscript; and decision to submit the manuscript for publication.

Competing interests None declared.

Ethics approval Research ethics board.

Provenance and peer review Not commissioned; externally peer reviewed.

Open Access This is an Open Access article distributed in accordance with the Creative Commons Attribution Non Commercial (CC BY-NC 4.0) license, which permits others to distribute, remix, adapt, build upon this work non-

commercially, and license their derivative works on different 
terms, provided the original work is properly cited and the use is non-commercial. See: http://creativecommons.org/licenses/by$\mathrm{nc} / 4.0 /$

\section{REFERENCES}

1 Wilson ML, Gaido L. Laboratory diagnosis of urinary tract infections in adult patients. Clin Infect Dis 2004;38:1150-8.

2 Nicolle LE, Bradley S, Colgan R, et al. Infectious Diseases Society of America guidelines for the diagnosis and treatment of asymptomatic bacteriuria in adults. Clin Infect Dis 2005;40:643-54.

3 Silver SA, Baillie L, Simor AE. Positive urine cultures: a major cause of inappropriate antimicrobial use in hospitals? Can J Infect Dis Med Microbiol 2009;20:107-11.

4 Leis JA, Gold WL, Daneman N, et al. Downstream impact of urine cultures ordered without indication at two acute care teaching hospitals. Infect Control Hosp Epidemiol 2013;34:1113-14.

5 Hartley SE, Kuhn L, Valley S, et al. Evaluating a hospitalist-based Intervention to decrease unnecessary antimicrobial use in patients with asymptomatic bacteriuria. Infect Control Hosp Epidemiol 2016;37:1044-51.

6 Walker S, McGeer A, Simor AE, et al. Why are antibiotics prescribed for asymptomatic bacteriuria in institutionalized elderly people?: a qualitative study of physicians' and nurses' perceptions. CMAJ 2000;163:273-7.

7 Rotjanapan P, Dosa D, Thomas KS. Potentially inappropriate treatment of urinary tract infections in two Rhode Island nursing homes. Arch Intern Med 2011;171:438-43.

8 Trautner BW, Petersen NJ, Hysong SJ, et al. Overtreatment of asymptomatic bacteriuria: identifying provider barriers to evidence-based care. Am J Infect Control 2014;42:653-8.

9 Jones CW, Culbreath KD, Mehrotra A, et al. Reflect urine culture cancellation in the emergency department. J Emerg Med 2014;46:71-6.
10 Chironda B, Clancy S, Powis JE. Optimizing urine culture collection in the emergency department using frontline ownership interventions. Clin Infect Dis 2014;59: 1038-9.

11 Loeb M, Brazil K, Lohfeld L, et al. Effect of a multifaceted intervention on number of antimicrobial prescriptions for suspected urinary tract infections in residents of nursing homes: cluster randomised controlled trial. $B M$ J 2005;331:669.

12 Bonnal C, Baune B, Mion M, et al. Bacteriuria in a geriatric hospital: impact of an antibiotic improvement program. J Am Med Dir Assoc 2008;9:605-9.

13 Linares LA, Thornton DJ, Strymish J, et al. Electronic memorandum decreases unnecessary antimicrobial use for asymptomatic bacteriuria and culture-negative pyuria. Infect Control Hosp Epidemiol 2011;32:644-8.

14 Leis JA, Rebick GW, Daneman N, et al. Reducing antimicrobial therapy for asymptomatic bacteriuria among noncatheterized inpatients: a proof-of-concept study. Clin Infect Dis 2014;58:980-3.

15 Eisinger SW, Schwartz M, Dam L, et al. Evaluation of the BD Vacutainer Plus Urine C\&S Preservative Tubes compared with nonpreservative urine samples stored at $4 \mathrm{C}$ and room temperature. Am J Clin Pathol 2013;140:306-13.

16 Christiaens TC, De Meyere M, Verschraegen G, et al. Randomised controlled trial of nitrofurantoin versus placebo in the treatment of uncomplicated urinary tract infection in adult women. Br J Gen Pract 2002;52:729-34.

17 Hertz JT, Lescallette RD, Barrett TW, et al. External validation of an ED protocol for reflex urine culture cancelation. Am J Emerg Med 2015;33:1838-9.

18 Dumkow LE, Kenney RM, MacDonald NC, et al. Impact of a multidisciplinary culture follow-up program of antimicrobial therapy in the emergency department. Infect Dis Ther 2014;3:45-53. 